\title{
Search for Lepton-Flavor-Violating Decays of Bosons With the ATLAS Detector
}

\author{
Tomas Davidek $^{1 *}$ and Luca Fiorini ${ }^{2 *}$ \\ ${ }^{1}$ Faculty of Mathematics and Physics, Institute of Particle and Nuclear Physics (IPNP), Charles University, Prague, Czechia, \\ ${ }^{2}$ Instituto de Física Corpuscular (IFIC), CSIC-Universitat de València, Valencia, Spain
}

The quest for lepton-flavor-violating processes at the LHC represents one of the key searches for new physics beyond the Standard Model. This review summarizes the direct searches for lepton-flavor-violating decays of heavy bosons with the ATLAS detector, using proton-proton collisions at the center-of-mass energy of $13 \mathrm{TeV}$.

Keywords: lepton-flavor-violating decays, $Z$ boson, Higgs boson, ATLAS, LHC, particle physics

\section{OPEN ACCESS}

Edited by:

María J. Herrero,

Autonomous University of Madrid,

Spain

Reviewed by:

Zhenbin Wu,

University of lllinois at Chicago,

United States

Rui Ribeiro Santos,

Instituto Superior de Engenharia de

Lisboa, Portugal

*Correspondence:

Tomas Davidek

tomas.davidek@cern.ch

Luca Fiorin

luca.fiorini@cern.ch

Specialty section:

This article was submitted to

High-Energy and Astroparticle

Physics,

a section of the journa

Frontiers in Physics

Received: 18 September 2019 Accepted: 15 April 2020 Published: 08 May 2020

Citation:

Davidek T and Fiorini L (2020) Search for Lepton-Flavor-Violating Decays of Bosons With the ATLAS Detector.

Front. Phys. 8:149

doi: 10.3389/fphy.2020.00149

\section{INTRODUCTION}

The search for processes beyond the Standard Model (SM) is one of the goals of the physics programme at the Large Hadron Collider (LHC) at CERN. A possible sign of such processes would be the lepton-flavor-violation (LFV) in decays of heavy bosons, such as well-established $Z$ and Higgs bosons or even not-yet-discovered bosons ( $Z^{\prime}$, additional Higgs bosons, etc).

Direct LFV processes are forbidden in the SM but are allowed in its many extensions. For instance, LFV decays of the $Z$ boson are predicted by models with heavy neutrinos [1], extended gauge models [2] and supersymmetry (SUSY) [3], which also allows for LFV decays of the Higgs boson $[4,5]$. In particular Minimal Supersymmetric SM scenarios with very high energy scale of the SUSY particle masses $m_{\text {SUSY }}$ present a non-decoupling behavior of the Higgs boson partial widths in two leptons of different flavor, with respect to $m_{\mathrm{SUSY}}$. This feature opens the possibility of detecting indirectly scenarios with very high $m_{\text {SUSY }}$ via LFV Higgs boson decays. Other models with more than one Higgs doublet [6,7], composite Higgs [8] or warped extra dimension models [9-11] predict LFV decays of the Higgs boson, too. The addition of an extra $U(1)$ gauge symmetry to the SM results in a massive neutral $Z^{\prime}$ boson [12] that could also decay through LFV processes. Recently, new models [13-15] have proposed LFV decays of the Higgs or heavier bosons as a necessary ingredient to explain the flavor anomalies observed by LHCb [16-21].

This paper summarizes the searches for LFV decays performed with the ATLAS detector using $p p$ collision data collected at the center-of-mass energy of $13 \mathrm{TeV}$. Despite the large number of aforementioned models predicting the existence of LFV processes, the ATLAS searches presented in this review are performed as much as possible in a model-independent way, in order to be sensitive to any type of new physics phenomena giving rise to LFV processes. First, the ATLAS detector and object selection common to below analyses are briefly described (section 2). The latest ATLAS results on LFV decays of $Z$, Higgs bosons and searches for other bosons decaying via LFV processes are summarized in sections 3,4 , and 5 , respectively.

\section{THE ATLAS DETECTOR AND OBJECT SELECTION}

\subsection{The ATLAS Detector}

ATLAS is a multi-purpose particle detector at the LHC with a forward-backward symmetry and nearly $4 \pi$ coverage in the solid angle [22-24]. It consists of several sub-detectors that play different 
roles. Closest to the beam pipe is the inner tracking detector covering the pseudorapidity ${ }^{1}$ range $|\eta|<2.5$. It is surrounded by the superconducting solenoid that provides a $2 \mathrm{~T}$ axial magnetic field. High-granularity electromagnetic $(|\eta|<3.2)$ and hadronic $(|\eta|<4.9)$ sampling calorimeters provide precision energy and direction measurement of electrons, photons, isolated hadrons and jets. The detector is completed with the muon spectrometer $(|\eta|<2.7)$, with a toroidal magnetic field generated by three sets of large superconducting magnets. The spectrometer also includes fast trigger chambers used for the event selection in the first trigger level.

\subsection{Object Selection}

The key objects used in these analyses include light leptons ${ }^{2}$, jets including those originating from hadronic decays of $\tau$-leptons and missing transverse momentum.

Electrons are reconstructed by matching tracks in the inner detector to clustered energy deposits in the electromagnetic calorimeter [25]. Likelihood-based identification (ID) [26] is applied. Muons are identified by mutually matching tracks in the inner detector and muon spectrometer [27]. Electrons and muons are required to be isolated from other objects reconstructed in the detector in order to suppress events where these leptons originate from semi-leptonic decays of particles within jets or from other sources.

Jets are reconstructed with the anti- $k_{t}$ algorithm [28] applied to topological clusters of calorimeter cells [29] with a radius parameter $R=0.4$. Jets from other $p p$ interactions in the same and neighboring bunch-crossings are mitigated with the jet vertex tagger algorithms $[30,31]$. Jets containing $b$-hadrons ( $b$-jets), often originating from top-quark decays, are identified with the likelihood-based algorithm [32,33] used in the central detector region $(|\eta|<2.4)$, which is inside the inner tracking detector coverage.

The reconstruction of visible products of the hadronic $\tau$ lepton decays $\left(\tau \rightarrow\right.$ hadrons $\left.+v_{\tau}\right)$, denoted $\tau_{\text {had-vis, }}$, starts from jets. Additional information from the inner detector tracks associated to the given jet is exploited, one or three tracks with a total charge $q= \pm 1$ are required. A dedicated identification algorithm $[34,35]$ is further applied in order to reject candidates arising from misidentified jets or from decays of hadrons containing $b$ - or $c$-quark. Finally, 1-prong $\tau_{\text {had-vis }}$ candidates geometrically overlapping with an electron with high ID score are also rejected. For each identified $\tau_{\text {had-vis }}$ candidate,
The missing transverse momentum $\vec{p}_{\mathrm{T}}^{\text {miss }}$ and its magnitude $E_{\mathrm{T}}^{\mathrm{miss}}$ is calculated as negative vectorial sum of the transverse momenta of all fully reconstructed and calibrated physics objects. Additional contribution comes from the tracks originating from the hard-scattering vertex that are not matched to any reconstructed object [36].

\subsection{Dilepton Mass Reconstruction}

In order to reconstruct a two-body decay of a heavy boson, the invariant mass of the decay products needs to be constructed. In case of the $e \mu$ decay, this quantity can be directly evaluated from individual measured lepton momenta. The situation is more complicated in decays involving $\tau$-leptons due to the presence of at least one neutrino in the final state. Two approaches are usually adopted, both exploiting $\vec{p}_{\mathrm{T}}^{\text {miss }}$ as a proxy to the neutrino momentum in the plane transverse to the colliding beam axis. The collinear approximation [37] assumes all $\tau$-decay products including the neutrino fly in the same direction, while the missing mass calculator (MMC) [37] allows for a non-zero angle between the neutrino and the direction of the visible $\tau$-decay products, taking into account the angular distribution expected for the given decay mode.

\section{3. $Z$ BOSON}

The searches for LFV decays of the $Z$ boson include all flavor combination $Z \rightarrow e \tau, Z \rightarrow \mu \tau$ and $Z \rightarrow e \mu$. This review focuses on the two searches with $\tau$-lepton involved, where the results with the $p p$ collisions acquired at the center-of-mass energy of $13 \mathrm{TeV}$ and corresponding to the total integrated luminosity of $36.1 \mathrm{fb}^{-1}$ are already available [38].

Major background processes to the $Z \rightarrow \ell \tau$ search include the irreducible component $Z \rightarrow \tau \tau \rightarrow \ell+$ hadrons $+3 v$ and reducible backgrounds from $W+$ jets, top-quark pair-production and $Z \rightarrow \ell \ell$, where either a jet or a light lepton is misidentified as $\tau_{\text {had-vis. }}$.

Neural network (NN) classifiers are used to identify the three major background components $(Z \rightarrow \tau \tau, W+$ jets, $Z \rightarrow \ell \ell)$. For each type the NN classifier is trained and validated with the MC simulations. Components of light lepton and $\tau_{\text {had-vis }}$ momenta, their collinear mass and missing transverse momentum are typically used as the $\mathrm{NN}$ input variables. In order to obtain a single discriminating quantity, the combined $\mathrm{NN}$ classifier is built of individual NN outputs separately for events with 1-prong and 3-prong $\tau_{\text {had-vis }}$ candidates:

$$
\begin{aligned}
\text { combined(1-prong) }= & 1-\sqrt{(1-\mathrm{NN}(W+\mathrm{jets}))^{2}+(1-\mathrm{NN}(Z \rightarrow \tau \tau))^{2}+(1-\mathrm{NN}(Z \rightarrow \ell \ell))^{2}} / \sqrt{3} \\
& \text { combined(3-prong) }=1-\sqrt{(1-\mathrm{NN}(W+\mathrm{jets}))^{2}+(1-\mathrm{NN}(Z \rightarrow \tau \tau))^{2}} / \sqrt{2}
\end{aligned}
$$

the corresponding jet is removed from the jet collection to avoid object double-counting.

\footnotetext{
${ }^{1}$ The pseudorapidity is defined as $\eta \equiv-\ln \tan (\theta / 2)$, where $\theta$ represents the polar angle measured from the beam axis.

${ }^{2}$ Electrons and muons are referred to as light leptons, denoted by $\ell$. All lepton flavors including $\tau$-lepton are denoted by $l$.
}

Events considered in the analysis are triggered by single electron or muon triggers. The selection is further tightened by requiring well-identified and isolated electron or muon matched to the triggered lepton. Only hadronic decays of the $\tau$-lepton are considered in the final state, at least one $\tau_{\text {had-vis }}$ candidate with opposite electric charge with respect to the light lepton 
is required. Events with more than one light lepton and/or $b$ jets are rejected, the latter condition mitigates the top-quarkrelated background. The transverse mass of the $\tau_{\text {had-vis }}$ candidate and $E_{\mathrm{T}}^{\mathrm{miss}}$ :

$$
m_{\mathrm{T}}\left(\tau_{\text {had-vis }}, E_{\mathrm{T}}^{\text {miss }}\right) \equiv \sqrt{2 p_{\mathrm{T}}\left(\tau_{\text {had-vis }}\right) E_{\mathrm{T}}^{\text {miss }}\left(1-\cos \left(\Delta \phi\left(\tau_{\text {had-vis }}, E_{\mathrm{T}}^{\text {miss }}\right)\right)\right)}
$$

also assists in the signal/background separation. ${ }^{3}$ In the signal region $(\mathrm{SR}), m_{\mathrm{T}}\left(\tau_{\text {had-vis }}, E_{\mathrm{T}}^{\text {miss }}\right)<35(30) \mathrm{GeV}$ in the $e \tau(\mu \tau)$ search is further required.

In order to constrain the background normalization with data, four control regions (CR) are defined for several background components. The event selection is the same as for the SR, but one criterion is inverted: at least two $b$-jets in the top-quark CR, $m_{\mathrm{T}}\left(\tau_{\text {had-vis }}, E_{\mathrm{T}}^{\mathrm{miss}}\right)>40 \mathrm{GeV}$ in the $W+$ jets $\mathrm{CR}$, inverted light lepton isolation in the multi-jet CR. Electrons misidentified as 1prong $\tau_{\text {had-vis }}$ are determined from MC simulations, the rate is corrected using data. Normalization is constrained from the $Z \rightarrow$ $\ell \ell \mathrm{CR}$, defined with dilepton invariant mass corresponding to the $Z$-boson mass. Jets misidentified as $\tau_{\text {had-vis }}$ are estimated using the data-driven fake factor method. The dominant contributions comes from $W+$ jets and multi-jet processes, the fake factors are measured in the corresponding CR.

The NN templates are constructed using the combined outputs (1), (2) for signal, $Z \rightarrow \tau \tau$, fakes, $Z \rightarrow \ell \ell$, top-quark background and $W(\rightarrow \tau \nu)+$ jets events; small contributions from SM Higgs boson decays and diboson production are summed into a single template ("other"). The template fit is performed separately for each search and prongness as shown in Figure 1. The dominant contributions to the total uncertainty come from the normalizations of the $Z \rightarrow \tau \tau$ background and components where jets mimic $\tau_{\text {had }}(W+$ jets, $\bar{t}, Z(\rightarrow$ $\ell \ell)+$ jets, multi-jets). The statistical uncertainty in fake factors also contributes significantly to the total uncertainty.

The combined best-fit value of the branching fraction slightly fluctuates to a positive value in the e $\tau$ search, $\mathcal{B}(Z \rightarrow e \tau)=$ $\left(3.3_{-1.4}^{+1.5}\right) \times 10^{-5}$, and it is consistent with zero in the $\mu \tau$ search, $\mathcal{B}(Z \rightarrow \mu \tau)=\left(-0.1_{-1.2}^{+1.2}\right) \times 10^{-5}$. Since no significant deviations from the background-only hypothesis are observed, the upper limits at $95 \%$ confidence level $(\mathrm{CL})$ are set, $\mathcal{B}(Z \rightarrow$ e $\tau)<$ $5.8 \times 10^{-5}$ and $\mathcal{B}(Z \rightarrow \mu \tau)<2.4 \times 10^{-5}$, respectively [38].

The results of the $Z \rightarrow \mu \tau$ search are combined with the previous results obtained with $p p$ collisions at the center-of-mass energy of $8 \mathrm{TeV}$ [39]. The combined observed 95\% CL upper limit amounts to $\mathcal{B}(Z \rightarrow \mu \tau)<1.3 \times 10^{-5}$.

The search for the LFV $Z \rightarrow e \mu$ decay was performed with earlier dataset acquired with $8 \mathrm{TeV} p p$ collisions. An upper bound $\mathcal{B}(Z \rightarrow e \mu)<7.5 \times 10^{-7}$ was obtained [40].

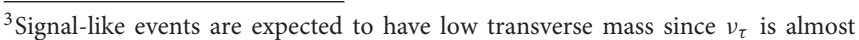
parallel to $\tau_{\text {had-vis }}$ due to large boost of the $\tau$-lepton, whereas $W+$ jets events exhibit large $m_{\mathrm{T}}\left(\tau_{\text {had-vis }}, E_{\mathrm{T}}^{\text {miss }}\right)$ values.
}

\section{HIGGS BOSON}

ATLAS and CMS collaborations discovered in 2012 a new boson with a mass of $\sim 125 \mathrm{GeV}[41,42]$. Current measurements indicate that the new particle is compatible with the predicted Higgs boson of the Standard Model (SM) [43-48], but the quest of measuring the branching fractions of the Higgs boson decay is still open. The search for LFV decays of the Higgs boson is performed for all flavor combinations: $H \rightarrow e \tau, H \rightarrow \mu \tau$, and $H \rightarrow e \mu$. The first two searches, involving decays with a $\tau$-lepton, are performed with the $p p$ collisions acquired at the center-of-mass energy of $13 \mathrm{TeV}$ and corresponding to a total integrated luminosity of $36.1 \mathrm{fb}^{-1}$ [49]. The last search is performed with $139 \mathrm{fb}^{-1}$ of $p p$ collisions at a center-of-mass energy of $13 \mathrm{TeV}$ [50].

\subsection{Search for $H \rightarrow e \tau$ and $H \rightarrow \mu \tau$ Decays}

Major background processes to the $H \rightarrow \ell \tau$ searches include the irreducible components $Z \rightarrow \tau \tau, H \rightarrow \tau \tau$ and reducible backgrounds from top-quark production processes, diboson production, $H \rightarrow W W$ and from $W+$ jets, $Z \rightarrow \ell \ell$ and multi-jet production where at least a jet or a light lepton is misidentified as $\tau_{\text {had-vis }}$ or light lepton of different nature.

The events considered in these two searches are triggered by single electron or muon triggers. The selection is further tightened by requiring well-identified and isolated electron or muon matched to the triggered lepton. For each of the two searches, two channels are exploited:

- $\ell \tau_{\ell^{\prime}}$ channel: requiring exactly one electron and one muon of opposite-sign (OS) charges, where the lepton of lowest $p_{\mathrm{T}}$ is associated to the leptonic decay of the $\tau$-lepton.

- $\ell \tau_{\text {had }}$ channel: requiring a light lepton and a $\tau_{\text {had-vis }}$ of OS charges.

For both channels, events are further categorized into VBF (with a focus on the Higgs boson production via the vector boson fusion, VBF) and non-VBF categories. The VBF selection is based on the kinematics of the two jets with the highest $p_{\mathrm{T}}$, where $\mathrm{j}_{1}$ and $\mathrm{j}_{2}$ denote the leading and subleading jet in $p_{\mathrm{T}}$, respectively. The variables $m\left(\mathrm{j}_{1}, \mathrm{j}_{2}\right)$ and $\Delta \eta\left(\mathrm{j}_{1}, \mathrm{j}_{2}\right)$ stand for the invariant mass and $\eta$ separation of these two jets. The non-VBF category contains events failing the VBF selection, but passing further selection criteria described in reference [49].

The VBF and non-VBF categories in each of the $\ell \tau_{\ell^{\prime}}$ and $\ell \tau_{\text {had }}$ channels give rise to the four signal regions used in the statistical analysis of each search. The analysis exploits boosted decision trees (BDT) [51-54] algorithms to enhance the signal separation from backgrounds in the individual searches, channels and categories. The analysis employs as well CRs orthogonal to the analysis SRs to constrain the background normalization with data. For each category, a top-quark control region is defined by requiring the presence of at least one $b$-jet. Similarly for each category, a $Z \rightarrow \tau \tau \mathrm{CR}$ is defined by requiring that the leading lepton $p_{\mathrm{T}}$ is between $35 \mathrm{GeV}$ and $45 \mathrm{GeV}$, with a purity of about $80 \%(65 \%)$ for the non-VBF (VBF) category.

The contribution of backgrounds where jets are misidentified as light leptons or $\tau_{\text {had-vis }}$ is estimated with data-driven 

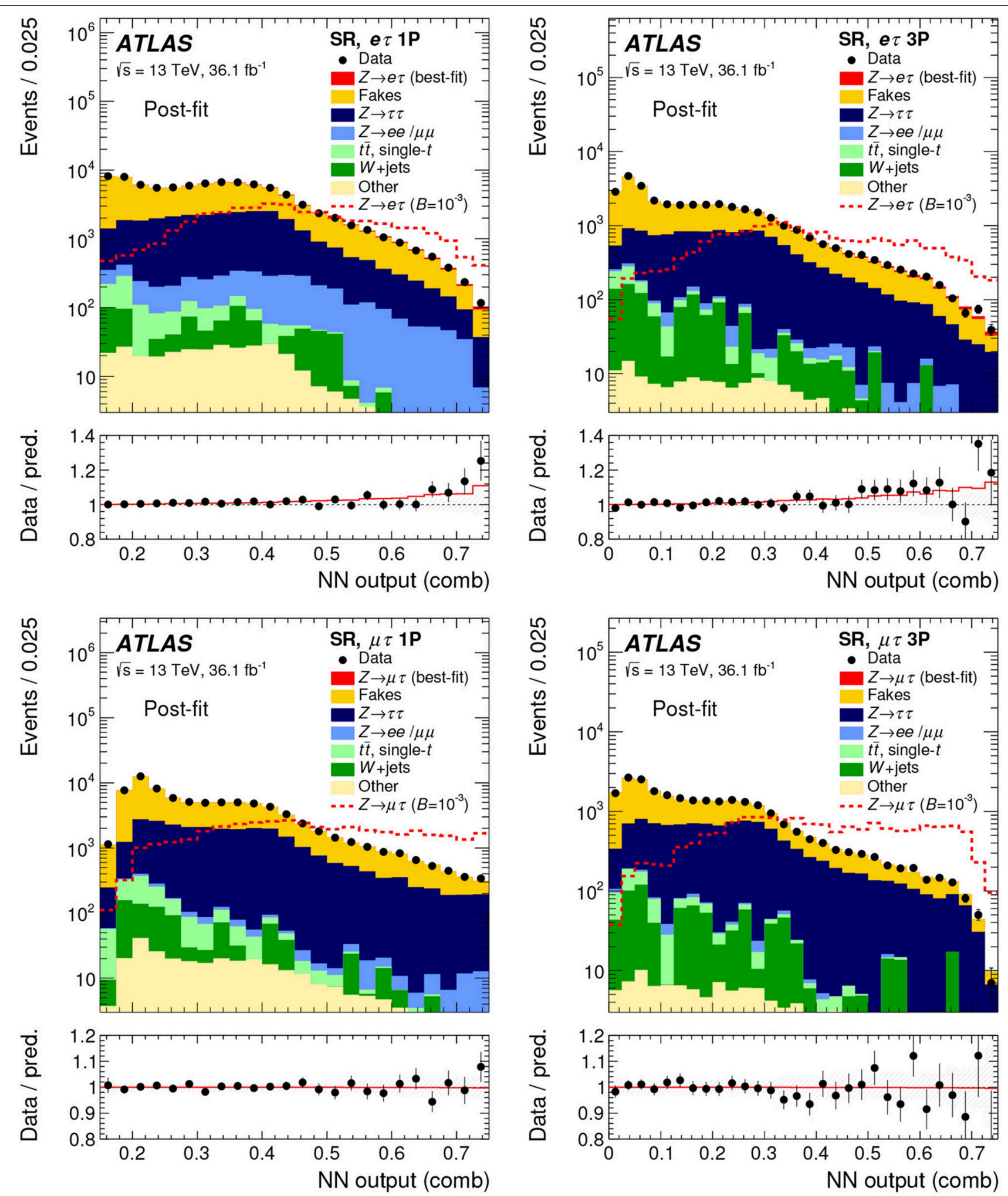

FIGURE 1 | Observed and expected post-fit distributions of the combined NN outputs in the SR are shown for the e $\tau$ (top row) and $\mu \tau$ (bottom row) searches, separately for 1-prong (left column) and 3-prong events (right column). The overlaid dashed line represents the expected distribution for the signal normalized to $\mathcal{B}(Z \rightarrow \ell \tau)=10^{-3}$. The bottom panels in each plot display the ratios of the observed data (dots) and the post-fit background plus signal (solid line) over the post-fit background. The hatched areas correspond to the combined statistical and systematic uncertainties. Reproduced from ATLAS Collaboration [38] under the Creative Commons CC-BY-4 license. 

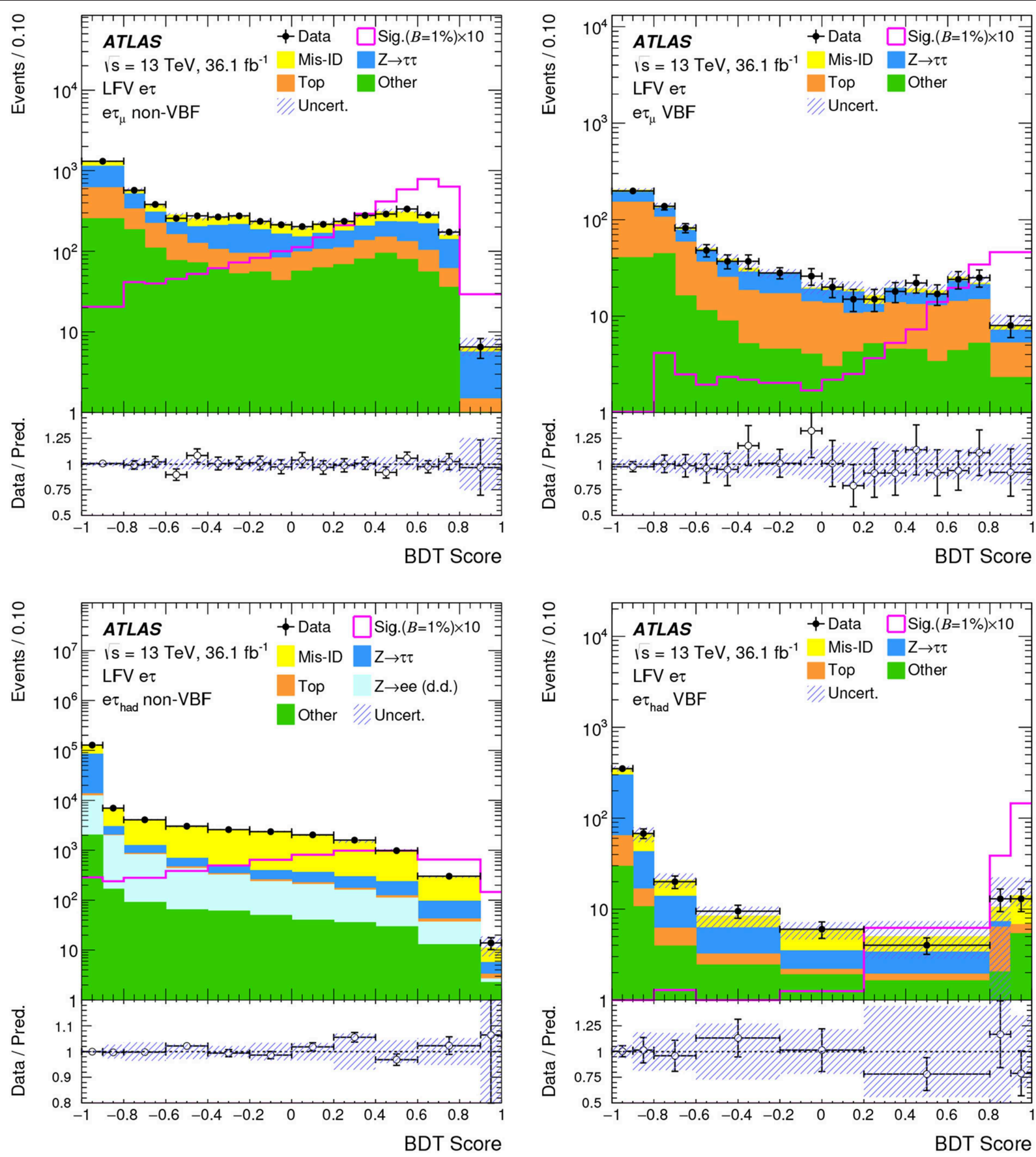

FIGURE 2 | Distributions of the BDT score after the background+signal fit in each signal region of the e $\tau$ search, with the LFV signal overlaid, normalized with $\mathcal{B}(H \rightarrow e \tau)=1 \%$ and enhanced by a factor 10 for visibility. The top and bottom plots display e $\tau_{\mu}$ and e $\tau_{\text {had }}$ BDT scores, respectively, the left (right) column corresponds to the non-VBF (VBF) category. The size of the combined statistical, experimental and theoretical uncertainties of the background is indicated by the hatched bands. The binning is shown as in the statistical analysis. Reproduced from ATLAS Collaboration [49] under the Creative Commons CC-BY-4 license.

techniques. Contribution of electrons misidentified as 1-prong $\tau_{\text {had-vis }}$ are determined from data, as well. The other background components are modeled with MC simulation.

The BDT distribution for the SRs of the $H \rightarrow e \tau$ and $H \rightarrow \mu \tau$ searches are shown in Figures 2, 3, respectively.
The main uncertainties of the searches derive from systematic uncertainties on the estimation of jets misidentified as $\tau_{\text {had-vis }}$ and light leptons and on the identification of $b$-jets. The bestfit branching fractions and upper limits are computed while assuming $\mathcal{B}(H \rightarrow \mu \tau)=0$ for the $H \rightarrow e \tau$ search and 

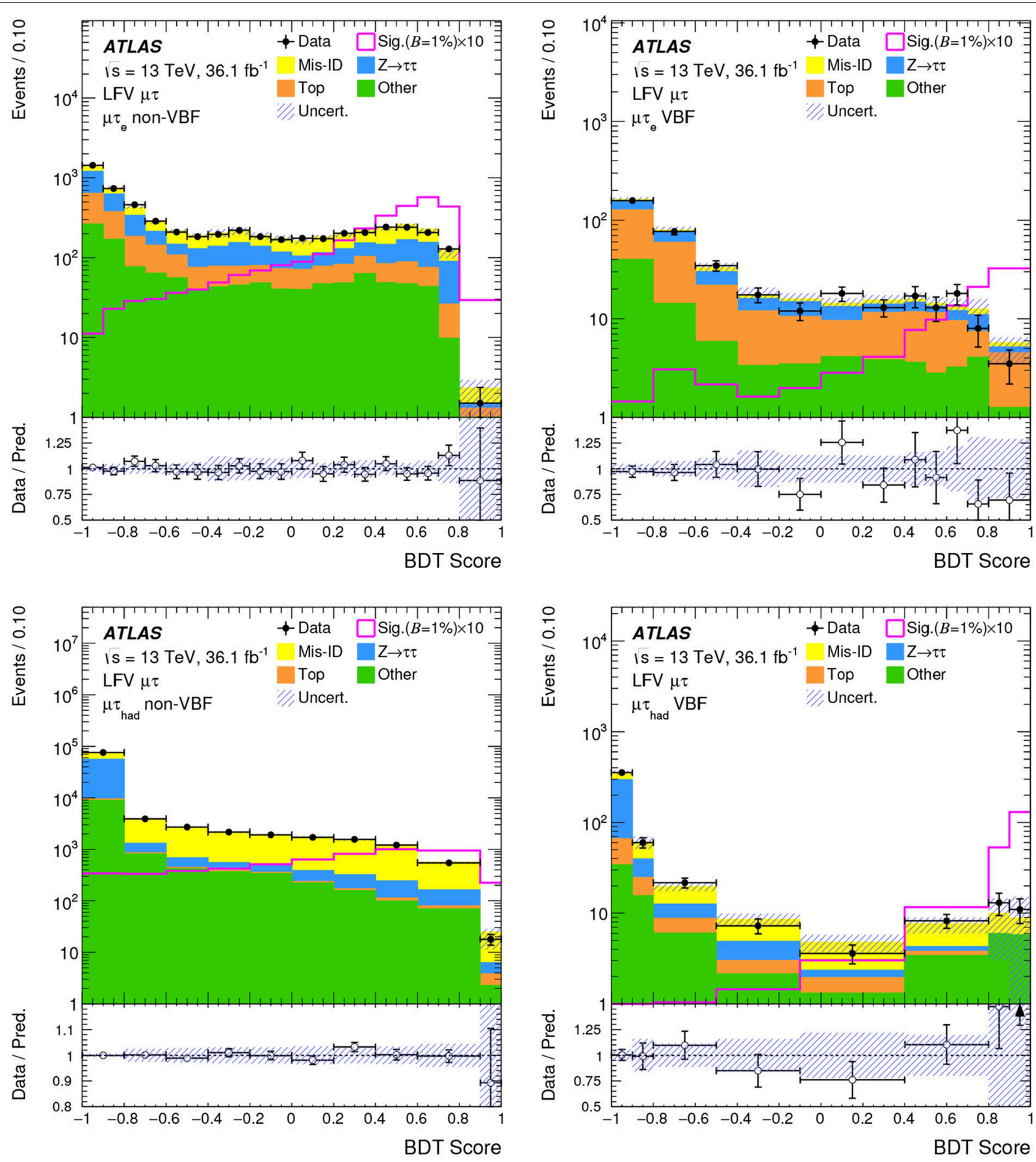

FIGURE 3 | Distributions of the BDT score after the background+signal fit in each signal region of the $\mu \tau$ search, with the LFV signal overlaid, normalized with $\mathcal{B}(H \rightarrow \mu \tau)=1 \%$ and enhanced by a factor 10 for visibility. The top and bottom plots display $\mu \tau_{e}$ and $\mu \tau_{\text {had }}$ BDT scores, respectively, the left (right) column corresponds to the non-VBF (VBF) category. The size of the combined statistical, experimental and theoretical uncertainties of the background is indicated by the hatched bands. The binning is shown as in the statistical analysis. In the data/background prediction ratio plots, points outside the displayed $y$-axis range are shown by arrows. Reproduced from ATLAS Collaboration [49] under the Creative Commons CC-BY-4 license.

$\mathcal{B}(H \rightarrow e \tau)=0$ for the $H \rightarrow \mu \tau$ search. The bestfit values of the LFV Higgs boson branching fractions are equal to $\left(0.15_{-0.17}^{+0.18}\right) \%$ and $(-0.22 \pm 0.19) \%$ for the $H \rightarrow$ $e \tau$ and $H \rightarrow \mu \tau$ search, respectively. In the absence of a significant excess above the expected background from SM processes, upper limits on the LFV branching fractions are set for a Higgs boson with $m_{H}=125 \mathrm{GeV}$. The observed (median expected) 95\% CL upper limits are $0.47 \%\left(0.34_{-0.10}^{+0.13} \%\right)$ and $0.28 \%\left(0.37_{-0.10}^{+0.14} \%\right)$ for the $H \rightarrow e \tau$ and $H \rightarrow \mu \tau$ searches, respectively. 

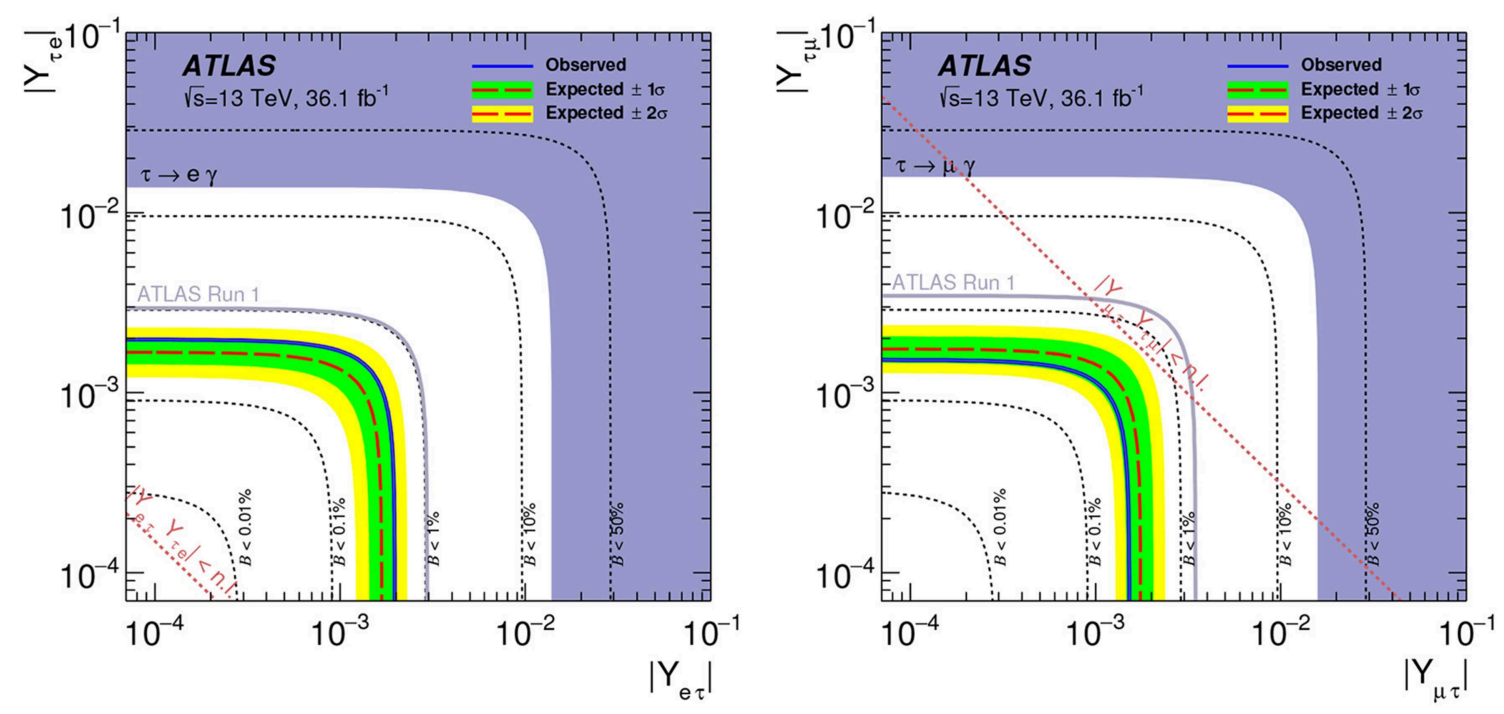

FIGURE 4 | Upper limits on the absolute value of the couplings $Y_{\tau \ell}$ and $Y_{\ell \tau}$ together with the limits from earlier ATLAS analyses [39, 57] (light gray line) and the most stringent indirect limits from $\tau \rightarrow \ell \gamma$ searches (dark purple region). Also indicated are limits corresponding to different branching fractions (0.01, $0.1,1,10$, and $50 \%$ ) and the naturalness limit (denoted n.I.) $\left|Y_{\tau \ell} Y_{\ell \tau}\right| \lesssim m_{\tau} m_{\ell} / v^{2}$ [55], where $v=246 \mathrm{GeV}$ is the vacuum expectation value of the Higgs field. Reproduced from ATLAS Collaboration [49] under the Creative Commons CC-BY-4 license.

The individual Yukawa matrix elements $Y_{\tau \ell}$ and $Y_{\ell \tau}$ are directly related to the corresponding branching fraction via the relation [55]

$$
\left|Y_{\ell \tau}\right|^{2}+\left|Y_{\tau \ell}\right|^{2}=8 \pi \frac{\Gamma_{H}^{S M}}{m_{H}} \cdot \frac{\mathcal{B}(H \rightarrow \ell \tau)}{1-\mathcal{B}(H \rightarrow \ell \tau)},
$$

where $m_{H}$ and $\Gamma_{H}^{S M}$ represent the mass and the SM total width of the Higgs boson [56]. Figure 4 shows the limits on the individual coupling matrix elements $Y_{\tau \ell}$ and $Y_{\ell \tau}$ together with the limits from earlier ATLAS analyses [39,57] and from $\tau \rightarrow \ell \gamma$ searches $[55,58]$.

\subsection{Search for $H \rightarrow e \mu$ Decay}

The analyses strategy follows closely the methodology of the $H \rightarrow \mu \mu$ search [59]. The main backgrounds of the search are $Z \rightarrow \tau \tau$ process, top-quark production, diboson production and $W+$ jets and multi-jet processes, where at least one jet is misidentified as a light lepton.

The signal is separated from the background primarily by identifying a narrow peak in the invariant mass distribution of the two leptons $m_{e \mu}$ corresponding to a decay of the Higgs boson with $m_{H}=125 \mathrm{GeV}$.

The event selection divides the events into eight categories, based on those used for the $H \rightarrow \mu \mu$ search. Events where the $p_{\mathrm{T}}$ of the subleading lepton is $<27 \mathrm{GeV}$ enter the "Low $p_{\mathrm{T}}$ " category. A category enriched in events from vector-boson fusion production "VBF" is defined by selecting those with jets having large pseudorapidity separation, $\Delta \eta\left(\mathrm{j}_{1}, \mathrm{j}_{2}\right)>3$ and dijet invariant mass $m\left(\mathrm{j}_{1}, \mathrm{j}_{2}\right)>500 \mathrm{GeV}$. Events that don't enter the first two categories are classified as "Central" if the pseudorapidities of both leptons are $|\eta|<1$ or as "Non-central" otherwise. For each of these two categories, events are further separated into three sub-categories depending on the ranges in the dilepton transverse momentum $p_{\mathrm{T}}(e \mu)$. Further details can be found in reference [50].

In the statistical analysis, analytical functions are used to describe the $m_{e \mu}$ distributions for the signal and background processes. The shape of the signal events in each category is parameterized as a sum of a Crystal Ball and a Gaussian function. For the background, a Bernstein polynomial function is used to parameterize the $m_{e \mu}$ distribution.

Distribution of the events entering in the eight categories used in the analysis is shown in Figure 5.

A simultaneous fit using a profile-likelihood-ratio test statistics is performed to the observed electron-muon-mass spectra divided into $50 m_{e \mu}$ bins in each of the eight categories. No evidence for the decay $H \rightarrow e \mu$ is observed with a best fit value of the branching fraction of $(0.4 \pm 2.9$ (stat) \pm 0.3 (syst) $) \times$ $10^{-5}$. The uncertainty is dominated by the data statistics, while the largest systematic contribution is from Higgs boson production cross-section uncertainty. The observed (median expected) upper limit at the $95 \%$ confidence level is found to be $6.1 \times 10^{-5}\left(5.8 \times 10^{-5}\right)$.

Figure 6 shows the limits on the individual coupling matrix elements $Y_{e \mu}$ and $Y_{\mu e}$ together with the indirect constraints derived from null searches for $\mu \rightarrow e \gamma$ [60], $\mu \rightarrow 3 e$ [61] and $\mu \rightarrow e$ conversions on gold nuclei [62].

Figure 7 summarizes the 95\% CL upper limits on the branching fractions of the Higgs boson to lepton-flavor-violating decays, $H \rightarrow e \tau, H \rightarrow \mu \tau$, and $H \rightarrow e \mu$.

\section{OTHER LFV SEARCHES}

\section{1. $Z^{\prime}$ Boson}

The searches summarized here assume the $Z^{\prime}$ boson has the same couplings to quarks and chiral structure as the SM $Z$ boson, while in the leptonic modes only the LFV decays are allowed. The searches for three flavor combinations $(e \mu, e \tau, \mu \tau)$ are performed 


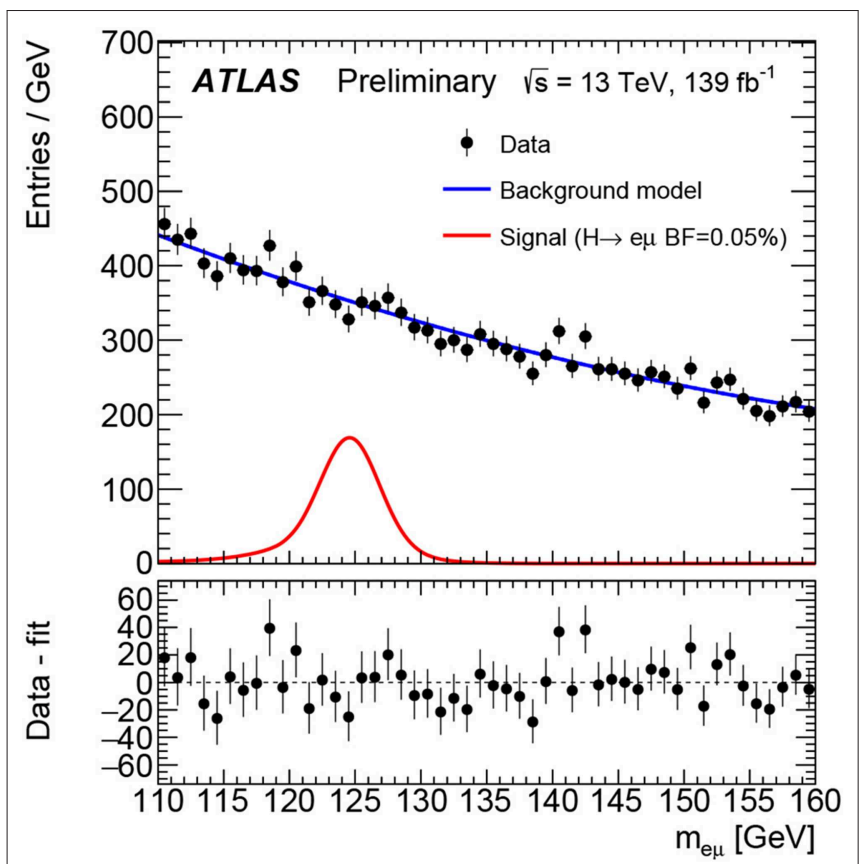

FIGURE 5 | All categories summed together for the e $\mu$ channel compared with the background only model. The signal parameterization with branching fractions (BF) set to $\mathcal{B}(H \rightarrow e \mu)=0.05 \%$ is also shown (red line). The bottom panels show the difference between data and fit. Reproduced from ATLAS Collaboration [50] under the Creative Commons CC-BY-4 license.

independently, assuming only one non-zero LFV coupling at any time [63].

The event selection is similar as in the analysis described in section 3 . In the $e \tau$ and $\mu \tau$ decays, only $\tau_{\text {had-vis }}$ candidates are considered. Higher $p_{\mathrm{T}}$ thresholds on all objects $\left(e, \mu, \tau_{\text {had-vis }}\right)$ are imposed, reflecting the high mass of the potential $Z^{\prime}$ boson. In addition, a tighter identification for light leptons is required. Exactly two back-to-back leptons (angular separation in the transverse plane $\left.\Delta \phi\left(l, l^{\prime}\right)>2.7\right)$ are required in the SR. No condition on the opposite charges is imposed, since it reduces the signal selection efficiency up to $6 \%$ at high masses due to charge misassignment, with no significant benefit in the background rejection.

The analysis strategy is to look for a peak in the invariant $(e \mu)$ or collinear $(e \tau, \mu \tau)$ mass distributions of the two decay products, commonly denoted as $m_{l l^{\prime}}$ in the following text. The irreducible background consists of $Z \rightarrow \tau \tau, t \bar{t}$, single topquark and diboson production processes, all of them are modeled with MC simulations. Since the top-quark samples $(t \bar{t}$ and single top-quark) suffer from low statistics for $m_{l l^{\prime}}>1 \mathrm{TeV}$, the $m_{l l^{\prime}}$ distribution is extrapolated with monotonically decreasing function fitted to that distribution in the lower mass range. The reducible background comes from jets misidentified as electrons or $\tau_{\text {had-vis }}$ candidates, it is estimated with data-driven techniques. Contributions from muons originating from hadron decays or jets is negligible and is not taken into account.

In the $e \mu$ search, the dominant background comes from the $t \bar{t}$ production, which can be suppressed by $b$-jet veto. The

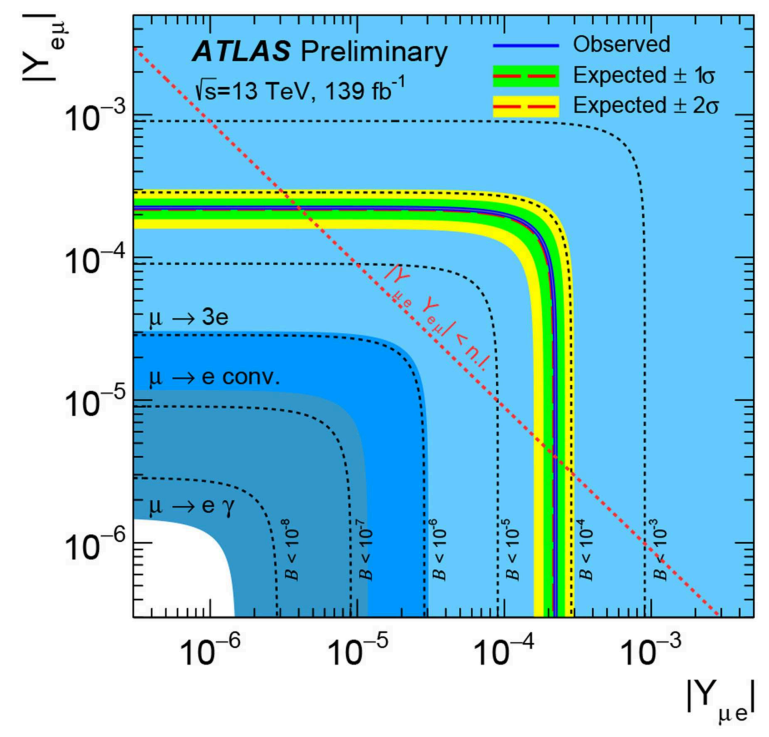

FIGURE 6 | Constraints on the flavor-violating Yukawa couplings $Y_{e \mu}$ and $Y_{\mu e}$ derived analogously to the relation (4). The expected (red dashed line) and observed (blue solid line) limits are derived from the limits on $\mathcal{B}(H \rightarrow e \mu)$ from the present analysis. The green (yellow) band indicates the range that is expected to contain 68\% (95\%) of all observed limit excursions. The shaded regions show the indirect constraints derived using the model calculations of reference [55] from null searches for $\mu \rightarrow$ e $\gamma$ [60], $\mu \rightarrow 3 e$ [61], and $\mu \rightarrow e$ conversions on gold nuclei [62]. For these calculations the flavor diagonal Yukawa couplings are taken to be the SM values. The diagonal line indicates the so-called naturalness limit $\left|Y_{e \mu} Y_{\mu e}\right|<m_{e} m_{\mu} / v^{2}$, where $v$ is the vacuum expectation value of the Higgs field. Reproduced from ATLAS Collaboration [50] under the Creative Commons CC-BY-4 license.

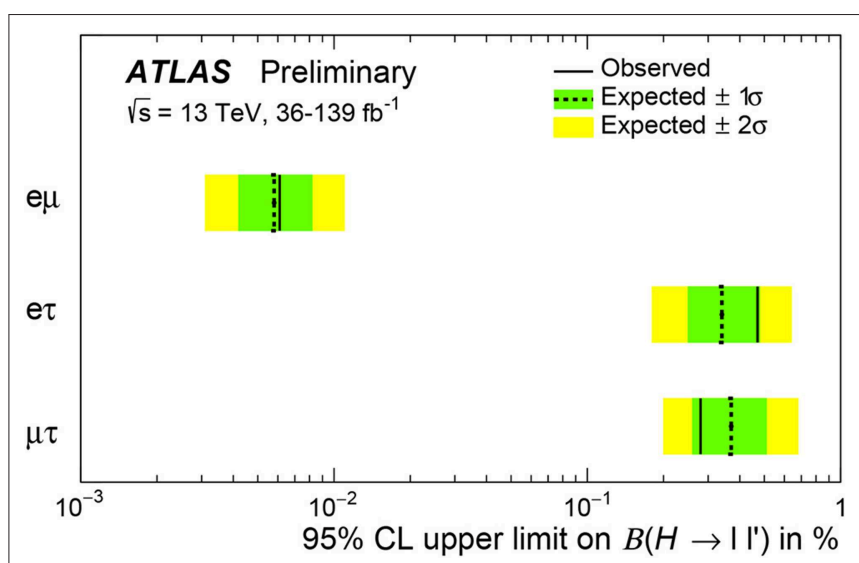

FIGURE 7 | Limits at 95\% CL upper limits on the branching fractions of the Higgs boson to various lepton flavor violating decays in percent. The results for $H \rightarrow e \mu$ are from reference [50] and the $H \rightarrow e \tau$ and $H \rightarrow \mu \tau$ results are taken from reference [49]. Expected limits are shown as a dashed line with the one- and two-sigma uncertainty bands in green and yellow. Reproduced from ATLAS Collaboration [50] under the Creative Commons CC-BY-4 license.

analysis is performed both with and without this requirement. The background from jets misidentified as electrons is estimated with the matrix method [64] using the samples with looser-ID electrons. Efficiencies of these electrons to match the nominal 

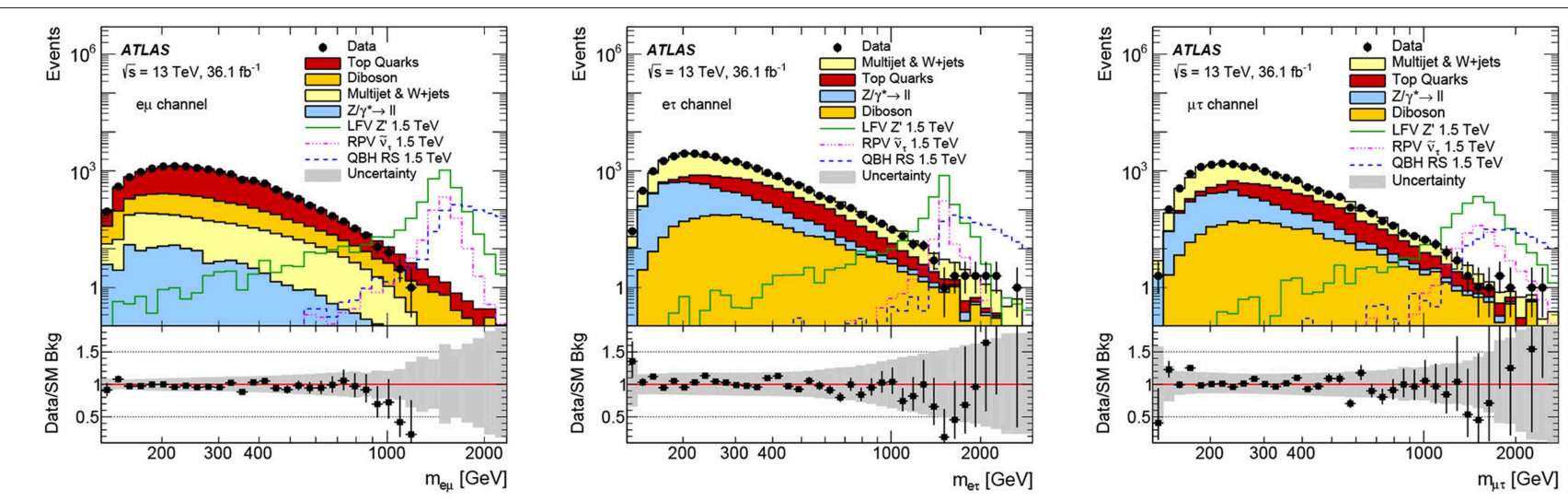

FIGURE 8 | The dilepton mass distributions of $e \mu$ without $b$-jet veto applied (left), e $\tau$ (middle), and $\mu \tau$ (right) pairs for data and SM predictions. The expected signal from $Z^{\prime}$ boson with a mass of $1.5 \mathrm{TeV}$ is overlaid. Also shown are signals expected by other models producing the same final states: $\tau$-sneutrino and quantum black hole, both with masses of $1.5 \mathrm{TeV}$. The error bars display the statistical uncertainty of the observed yields, the bands in the bottom panels include all statistical and systematic uncertainties combined in quadrature. No further data points are found in overflow bins. Reproduced from ATLAS Collaboration [63] under the Creative Commons CC-BY-4 license.
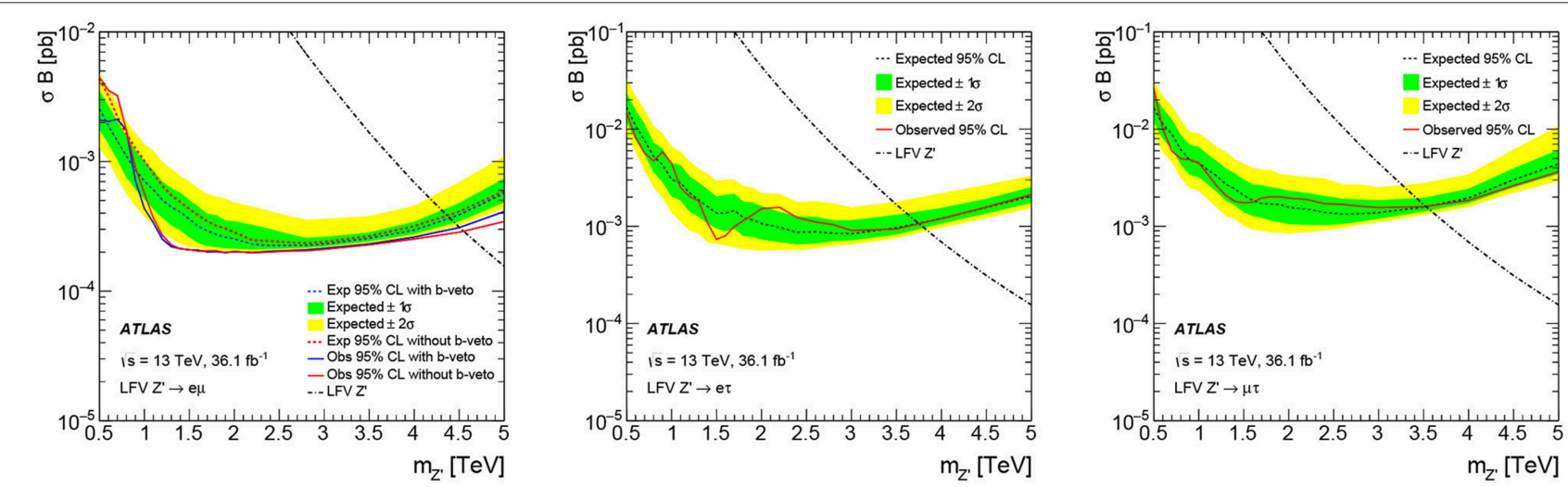

FIGURE 9 | The observed and expected 95\% CL upper limits on the $Z^{\prime}$ boson production cross-section times branching fraction for decays into e $\mu$ (left), $e \tau$ (middle), and $\mu \tau$ (right). The signal theoretical cross-section times branching fraction, shown with dash-dotted lines, are obtained from MC simulations according to the LFV $Z^{\prime}$ model $\left(\mathcal{B}\left(Z^{\prime} \rightarrow I I^{\prime}\right)=6.4 \%\right)$. The expected limits are shown with \pm 1 and \pm 2 standard deviation uncertainty bands. Reproduced from ATLAS Collaboration [63] under the Creative Commons CC-BY-4 license.

ones is evaluated with $Z \rightarrow$ ee simulated events, the misidentification probability (fake rate) is obtained in the multijet enriched data sample.

In the $e \tau$ and $\mu \tau$ searches, the dominant background comes from $W+$ jets process, where a jet is misidentified as a $\tau_{\text {had-vis }}$ candidate. It is estimated with MC simulations, where each jet is weighted according to the probability to pass the nominal $\tau$ lepton ID. This so-called fake rate is measured in the $W(\rightarrow$ $e / \mu)+$ jets $\mathrm{CR}$, defined in the same way as the SR but with the inverted condition on the back-to-back leptons. A cut on transverse mass, as defined in formula (3), is also required $\left(m_{\mathrm{T}}>\right.$ $80 \mathrm{GeV}$ ) to enrich this $\mathrm{CR}$ with $W+$ jets contribution. The fake rate is measured as a function of $p_{\mathrm{T}}, \eta$ and number of charged tracks associated with $\tau_{\text {had-vis }}$ candidates. Another source of reducible background is the multi-jet production. It is estimated with an ABCD method. Transfer factors are determined as the ratio of number of events failing both the light lepton isolation and $\tau$-lepton ID over the number of events passing these two criteria. These transfer factors are derived with the same-sign (SS) light lepton pairs, validated with opposite-sign events and applied to events without any charge requirement.

The dilepton mass spectra are displayed in Figure 8. The systematic uncertainties in parton distribution functions (PDFs) and the $t \bar{t}$ modeling represent the major contributions to the total uncertainty shown in the bottom panels. These $m_{l l^{\prime}}$ spectra are fitted with the BumpHunter program [65] to search for a possible peak. Since no significant deviations from the SM predictions are observed, the exclusion limits are extracted using a Bayesian method. The product of the Poisson probabilities of the observed and expected number of events in each $m_{l} l^{\prime}$ bin is used to construct the likelihood function. The 95\% CL exclusion upper limits are set on the signal cross-section times branching fraction. Expected exclusion limits are obtained by generating 1,000 pseudo-experiments for each mass point, their median value is taken as the expected limit. 


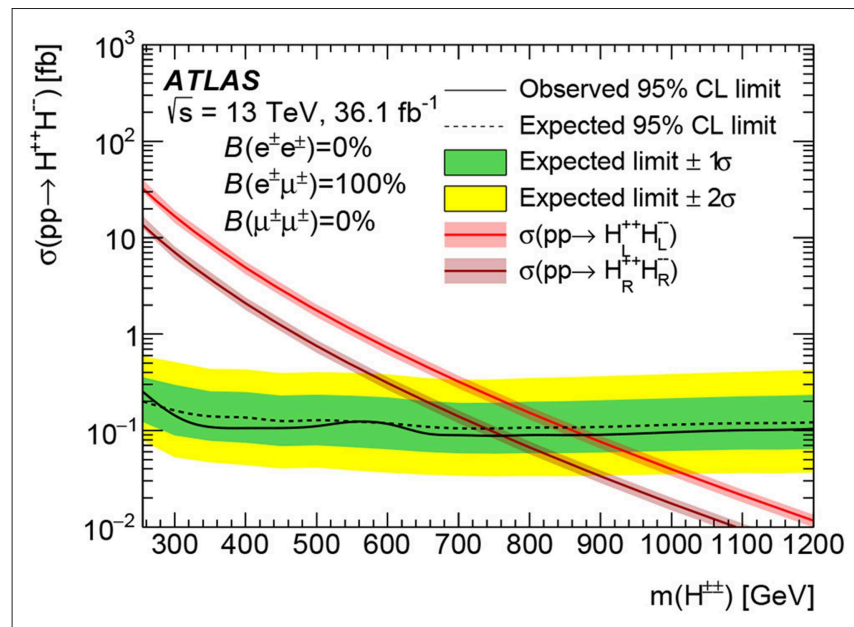

FIGURE 10 | The upper limit on the cross-section for $\mathrm{pp} \rightarrow \mathrm{H}^{++} \mathrm{H}^{--}$, assuming the doubly charged Higgs decays to a pair of electron and muon. The theoretical uncertainty in the cross-section is presented with the shaded band around the central value. Reproduced from ATLAS Collaboration [75] under the Creative Commons CC-BY-4 license.

The results are shown in Figure 9 as a function of the $Z^{\prime}$ boson mass for all three searches. The limits are not so strong for masses above $2.5 \mathrm{TeV}$ due to lower detector acceptance at very high $p_{\mathrm{T}}$, while in the low-mass region the limits suffer from the signal being suppressed by PDFs. The observed 95\% lower limits on the mass of the $Z^{\prime}$ boson with LFV couplings are $4.5 \mathrm{TeV}(4.4 \mathrm{TeV}$ when $b$-jet veto is applied), $3.7 \mathrm{TeV}$ and $3.5 \mathrm{TeV}$ in the $e \mu$, $e \tau$ and $\mu \tau$ channel, respectively.

The limits on couplings in the LFV $Z^{\prime}$ model are directly related to the cross-section times branching fraction. These limits can be then compared to results from precision low-energy measurements, namely to the $\mu$-to-e conversion, $\mu \rightarrow$ eee,

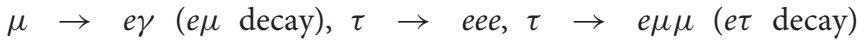
and $\tau \rightarrow \mu \mu \mu, \tau \rightarrow e \mu \mu$ ( $\mu \tau$ decay). The ATLAS results do not compete for the $e \mu$ decay, but are more stringent than the low-energy measurements for the $e \tau$ and $\mu \tau$ decays, although additional assumption on the $Z^{\prime}$ couplings to quarks are required [63].

The results on the presented searches are also interpreted in other models. Lower limits at 95\% CL are set on the $\tau$-sneutrino mass in the R-parity violating SUSY model [66] at 3.4, 2.9 and $2.6 \mathrm{TeV}$ and on the threshold mass for the quantum black hole production in the context of the Arkani-Hamed-DimopoulosDvali [67] (Randall-Sundrum [68]) model at 5.6 (3.4), 4.9 (2.9), and 4.5 (2.6) TeV for the $e \mu, e \tau$, and $\mu \tau$ search, respectively [63].

\subsection{Doubly Charged Higgs Boson}

Several extensions of the SM predict the existence of doubly charged Higgs boson $\left(H^{ \pm \pm}\right)$, for instance the Higgs triplet model [69], left-right symmetric (LRS) [70, 71], type-II seesaw $[72,73]$, and other models.

Doubly charged Higgs particles can couple to same-sign leptons (either left- or right-handed) or to pairs of $W$ bosons. The leptonic decay modes dominate for a low Higgs triplet vacuum expectation value $v_{\Delta}$, assumed in this search, which implies the main production mechanism at hadron colliders is the pair production $\mathrm{H}^{++} \mathrm{H}^{--}$via the Drell-Yan process. In the LRS models, the left- and right-handed cases are distinguished and referred to as $H_{\mathrm{L}}^{ \pm \pm}, H_{\mathrm{R}}^{ \pm \pm}$. The production cross-section for $\mathrm{H}_{\mathrm{L}}^{++} \mathrm{H}_{\mathrm{L}}^{--}$is $\sim 2.3$ times larger than for $\mathrm{H}_{\mathrm{R}}^{++} \mathrm{H}_{\mathrm{R}}^{--}$due to different couplings to $Z$ boson [74].

Unlike the SM Higgs boson, the doubly charged Higgs particle's couplings to fermions are not proportional to the corresponding fermion mass, therefore decays involving $\tau$ leptons are not preferred over those containing light leptons. For this reason, this review focuses on the LFV search for the $H^{ \pm \pm} \rightarrow e^{ \pm} \mu^{ \pm}$decay mode [75].

Events considered in the presented analysis are triggered by either combined $e-\mu$ trigger or single-lepton triggers. Only wellidentified, isolated and prompt ${ }^{4}$ light leptons are considered. At least one SS lepton pair with the invariant mass $m_{e \mu}>200 \mathrm{GeV}$ is required. In order to reduce the background originating from top-quark production processes, $b$-jet veto is applied. Three SRs are defined according to the number of light leptons $(2,3,4)$ in the final state. This choice also covers other final states of one of the $H^{ \pm \pm}$decay, such as $\tau$-lepton, $W$ or particles escaping detection. In the three- and four-lepton SRs, events are vetoed if any OS lepton pair exhibits an invariant mass close to that of the $Z$ boson. This condition mitigates the contribution from the diboson background. Additional requirements are imposed on the SS lepton pair in order to maximize the sensitivity of the search. In the two- and three-lepton SRs, the SS lepton pair must meet the criteria on the angular separation $\Delta R\left(e^{ \pm} \mu^{ \pm}\right)<3.5$, the total transverse momentum $p_{\mathrm{T}}\left(e^{ \pm} \mu^{ \pm}\right)>100 \mathrm{GeV}$ and the scalar sum of individual transverse momenta $p_{\mathrm{T}}\left(e^{ \pm}\right)+p_{\mathrm{T}}\left(\mu^{ \pm}\right)>$ $300 \mathrm{GeV}$. These criteria exploit both the boosted decay topology of the $H^{ \pm \pm}$resonance as well as its high mass translating into high energy of its decay products. In the four-lepton SR, events are rejected if the two SS lepton pairs exhibit large difference in their invariant masses.

The irreducible background consists of Drell-Yan, diboson and top-quark production, where the $Z$ boson, $W$ boson or the $\tau$-lepton from the $Z \rightarrow \tau \tau$ process decays to light leptons. Also the SM Higgs boson decays are considered. All these background components are simulated with MC.

The background originating from in-flight decays of mesons inside jets, jets misreconstructed as electrons, and conversions of initial- and final-state radiation photons is estimated with data using the fake factor method [76]. Another background component comes from the electron charge misidentification. Since this feature is poorly modeled by MC, data-driven approach is exploited using $Z \rightarrow$ ee events selected by imposing the di-electron invariant mass to match the $Z$ boson mass. The correction factors, derived as a function of electron's $p_{\mathrm{T}}$ and $\eta$ by comparing the number of SS and OS events between data and MC, are then applied to MC [75].

The statistical analysis exploits the simultaneous maximumlikelihood fit applied to mass distributions in all SRs and CRs. The SS dilepton mass is used in the two- and three-lepton SRs,

${ }^{4}$ Longitudinal impact parameter with respect to the primary vertex of $<0.5 \mathrm{~mm}$. 

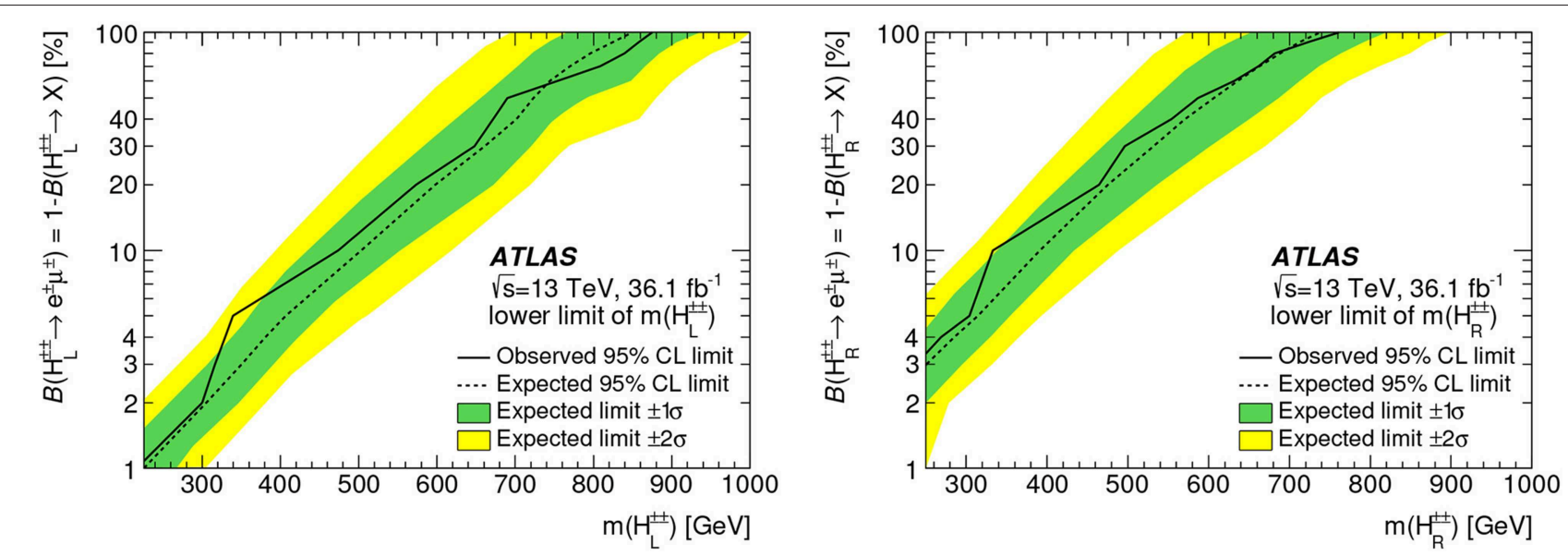

FIGURE 11 | Lower limits on the $H_{\mathrm{L}}^{ \pm \pm}$(left) and $H_{\mathrm{R}}^{ \pm \pm}$(right) boson mass as a function of the branching fraction $\mathcal{B}\left(H^{ \pm \pm} \rightarrow \mathrm{e}^{ \pm} \mu^{ \pm}\right.$). Reproduced from ATLAS Collaboration [75] under the Creative Commons CC-BY-4 license.

TABLE 1 | Current ATLAS limits on the branching fractions for the LFV decays of $Z$ and Higgs bosons, as obtained at $95 \%$ of $C L$, along with the corresponding dataset.

\begin{tabular}{lccc}
\hline Process & Current limit & Dataset & HL-LHC prospects \\
\hline$Z \rightarrow e \mu$ & $\mathcal{B}<7.5 \times 10^{-7}$ & $8 \mathrm{TeV}, 20 \mathrm{fb}^{-1}$ & \\
$Z \rightarrow e \tau$ & $\mathcal{B}<5.8 \times 10^{-5}$ & $13 \mathrm{TeV}, 36 \mathrm{fb}^{-1}$ & $\mathcal{B} \ll 9.8 \times 10^{-6}$ \\
$Z \rightarrow \mu \tau$ & $\mathcal{B}<1.3 \times 10^{-5}$ & $8 \mathrm{TeV}, 20 \mathrm{fb}^{-1}$ and & $\mathcal{B} \ll 1.2 \times 10^{-5}$ \\
& & $13 \mathrm{TeV}, 36 \mathrm{fb}^{-1}$ & \\
$H \rightarrow e \mu$ & $\mathcal{B}<6.1 \times 10^{-5}$ & $13 \mathrm{TeV}, 139 \mathrm{fb}^{-1}$ & \\
$H \rightarrow e \tau$ & $\mathcal{B}<0.47 \%$ & $13 \mathrm{TeV}, 36 \mathrm{fb}^{-1}$ & $\mathcal{B} \lesssim 0.05 \%$ \\
$H \rightarrow \mu \tau$ & $\mathcal{B}<0.28 \%$ & $13 \mathrm{TeV}, 36 \mathrm{fb}^{-1}$ & $\mathcal{B} \lesssim 0.05 \%$ \\
\hline
\end{tabular}

Limits expected from analyses of 3,000 $\mathrm{fb}^{-1}$ of pp collision data at HL-LHC are also given [77, 78], the symbol "«" indicates that limits are expected better than those obtained by LEP experiments [79-82].

while the average mass of the two SS dilepton pairs is used in the four-lepton SR. The signal is modeled with 23 templates of different $H^{ \pm \pm}$mass, ranging from 200 to $1300 \mathrm{GeV}$ in steps of $50 \mathrm{GeV}$. The main uncertainties of the search come from the systematic uncertainties on the fake background determination, theoretical uncertainties and statistical uncertainties of both data and MC samples. Since no evidence for the LFV decay of the doubly charged Higgs boson is observed, the cross-section upper limits are presented in Figure $\mathbf{1 0}$ as a function of the $H^{ \pm \pm}$ boson mass. The results of the fit for $H_{\mathrm{L}}^{ \pm \pm}$and $H_{\mathrm{R}}^{ \pm \pm}$are shown in Figure 11.

Similar analyses are performed also for the same-flavor lepton pairs $e^{ \pm} e^{ \pm}, \mu^{ \pm} \mu^{ \pm}$in the final state. Together with the presented search, lower limits on the mass of the $H_{\mathrm{L}}^{ \pm \pm}$boson are set, varying from 770 to $870 \mathrm{GeV}$ for $\mathcal{B}\left(H_{\mathrm{L}}^{ \pm \pm} \rightarrow \ell^{ \pm} \ell^{ \pm}\right)=100 \%$ [75].

\section{CONCLUSIONS AND OUTLOOK}

Direct searches for lepton-flavor-violating decays of heavy bosons, as performed with the ATLAS detector using $13 \mathrm{TeV}$ proton-proton collisions, have been presented. Since no signs of the LFV processes are observed, upper limits at 95\% CL are set on the corresponding branching fractions of the $Z$ and Higgs bosons. They are summarized in Table 1. Some of these results will soon be updated after the analyses of the full Run-2 dataset are completed. Further improvements are expected from the highluminosity LHC (HL-LHC), which should provide $3000 \mathrm{fb}^{-1}$ of $p p$ collisions at the center-of-mass energy of $14 \mathrm{TeV}$. The HLLHC results will provide the best limits on LFV decays of the $Z$ boson [77], overcoming the currently leading limits set by LEP experiments $\left(\mathcal{B}(Z \rightarrow e \tau)<9.8 \times 10^{-6}, \mathcal{B}(Z \rightarrow \mu \tau)<\right.$ $\left.1.2 \times 10^{-5}\right)$ at $95 \% \mathrm{CL}$ [79-82]. The limits on LFV $H \rightarrow \ell \tau$ decays have also been estimated [78] and are listed in Table $\mathbf{1 .}$

Searches for LFV decays of hypothetical new bosons predicted by various extensions of the Standard Model have also been performed and lower limits on their masses are set. The presented results are also interpreted in terms of other models, lower limits are set on the mass of the supersymmetric $\tau$-neutrino and the threshold mass for the quantum black hole production. Projections for HL-LHC also exist, for instance the LFV decays of doubly charged Higgs boson in the process $p p \rightarrow H^{++} H^{--} \rightarrow$ $\tau_{\text {had }}^{ \pm} \ell^{ \pm} \ell^{\mp} \ell^{\mp}$ can be probed up to $m\left(H^{ \pm \pm}\right) \sim 800 \mathrm{GeV}$ at the $3 \sigma$ CL [83].

\section{AUTHOR CONTRIBUTIONS}

The authors contributed to the review and summary of the current status of searches for lepton-flavor-violating decays in the ATLAS experiment. They also actively contributed to the ATLAS lepton-flavor-violating analyses.

\section{FUNDING}

The work was partly supported by the projects LTT17018 and LM2018104 of the Ministry of Education, Youth and Sports of the Czech Republic and by project RTI2018-094270-B-I00, financed by Spanish Ministry of Research and Universities, Spanish National Research Agency, and European Regional Development Fund (MCIU/AEI/ERDF, UE). 


\section{REFERENCES}

1. llana JI, Riemann T. Charged lepton flavor violation from massive neutrinos in Z decays. Phys Rev D. (2001) 63:053004. doi: 10.1103/PhysRevD.63.053004

2. Kuo TK, Nakagawa N. Lepton-flavor-violating decays of $Z^{0}$ and $\tau$. Phys Rev D. (1985) 32:306-7. doi: 10.1103/PhysRevD.32.306

3. Gabbiani F, Kim JH, Masiero A. $Z^{0} \rightarrow b \bar{s}$ and $Z^{0} \rightarrow \tau \mu$ in SUSY: are they observable? Phys Lett B. (1988) 214:398-402. doi: 10.1016/0370-2693(88)91384-6

4. Arana-Catania M, Arganda E, Herrero MJ. Non-decoupling SUSY in LFV Higgs decays: a window to new physics at the LHC. JHEP. (2013) 1309:160. doi: 10.1007/JHEP10(2015)192

5. Arhrib A, Cheng Y, Kong OCW. Comprehensive analysis on lepton flavor violating Higgs boson to $\mu^{\mp} \tau^{ \pm}$decay in supersymmetry without $R$ parity. Phys Rev D. (2013) 87:015025. doi: 10.1103/PhysRevD.87.015025

6. Bjorken JD, Weinberg S. Mechanism for Nonconservation of Muon Number. Phys Rev Lett. (1977) 38:622. doi: 10.1103/PhysRevLett.38.622

7. Diaz-Cruz JL, Toscano JJ. Lepton flavor violating decays of Higgs bosons beyond the standard model. Phys Rev D. (2000) 62:116005. doi: 10.1103/PhysRevD.62.116005

8. Agashe K, Contino R. Composite Higgs-mediated flavor-changing neutral current. Phys Rev D. (2009) 80:075016. doi: 10.1103/PhysRevD.80.075016

9. Perez G, Randall L. Natural neutrino masses and mixings from warped geometry. JHEP. (2009) 1:077. doi: 10.1088/1126-6708/2009/01/077

10. Azatov A, Toharia $M$, Zhu L. Higgs mediated flavor changing neutral current in warped extra dimensions. Phys Rev D. (2009) 80:035016. doi: 10.1103/PhysRevD.80.035016

11. Albrecht ME, Blanke M, Buras AJ, Duling B, Gemmler K. Electroweak and flavour structure of a warped extra dimension with custodial protection. JHEP. (2009) 9:064. doi: 10.1088/1126-6708/2009/09/064

12. Langacker P. The physics of heavy $Z^{\prime}$ Gauge Bosons. Rev Mod Phys. (2009) 81:1199-228. doi: 10.1103/RevModPhys.81.1199

13. Ko P, Omura Y, Shigekami Y, Yu C. LHCb anomaly and B physics in flavored $Z^{\prime}$ models with flavored Higgs doublets. Phys Rev D. (2017) 95:115040. doi: 10.1103/PhysRevD.95.115040

14. Carmona A, Goertz F. Lepton flavor and nonuniversality from minimal composite Higgs setups. Phys Rev Lett. (2016) 116:251801. doi: 10.1103/PhysRevLett.116.251801

15. Botella FJ, Branco GC, Nebot M, Rebelo MN. Flavour changing Higgs couplings in a class of two Higgs doublet models. Eur Phys J C. (2016) 76:161. doi: 10.1140/epjc/s10052-016-3993-0

16. Aaij R. Measurement of the ratio of branching fractions $\mathcal{B}\left(\bar{B}^{0} \rightarrow\right.$ $\left.D^{*}+\tau^{-} \bar{v}_{\tau}\right) / \mathcal{B}\left(\bar{B}^{0} \rightarrow D^{*}+\mu^{-} \bar{v}_{\mu}\right)$. Phys Rev Lett. (2015) $115: 111803$. doi: $10.1103 /$ PhysRevLett.115.111803

17. Aaij R. Measurement of the ratio of the $B^{0} \rightarrow D^{*}-\tau^{+} v_{\tau}$ and $B^{0} \rightarrow$ $D^{*}-\mu^{+} v_{\mu}$ branching fractions using three-prong $\tau$-lepton decays. Phys Rev Lett. (2018) 120:171802. doi: 10.1103/PhysRevLett.120.171802

18. Aaij R. Differential branching fractions and isospin asymmetries of $B \rightarrow$ $\left.K^{(} *\right) \mu^{+} \mu^{-}$decays. JHEP. (2014) 6:133. doi: 10.1007/JHEP06(2014)133

19. Aaij R. Angular analysis and differential branching fraction of the decay $B_{s}^{0} \rightarrow \phi \mu^{+} \mu^{-}$. JHEP. (2015) 9:179. doi: 10.1007/JHEP09(2015)179

20. Aaij R. Angular analysis of the $B^{0} \rightarrow K^{*} 0 \mu^{+} \mu^{-}$decay using $3 \mathrm{fb}^{-} 1$ of integrated luminosity. JHEP. (2016) 2:104. doi: 10.1007/JHEP02(2016)104

21. Aaij R. Differential branching fraction and angular analysis of $\Lambda_{b}^{0} \rightarrow \Lambda \mu^{+} \mu^{-}$ decays. JHEP. (2015) 6:115. doi: 10.1007/JHEP06(2015)115

22. ATLAS Collaboration. The ATLAS experiment at the CERN large Hadron collider. JINST. (2008) 3:S08003. doi: 10.1088/1748-0221/3/08/S08003

23. ATLAS Collaboration. ATLAS Insertable B-Layer Technical Design Report. ATLAS-TDR-19 (2010). Available online at: https://cds.cern.ch/record/ 1291633

24. Abbott B, Albert J, Alberti F, Alex M, Alimonti G, Alkire S, et al. Production and integration of the ATLAS insertable B-layer. JINST. (2018) 13:T05008. doi: 10.1088/1748-0221/13/05/T05008

25. ATLAS Collaboration. Electron and photon energy calibration with the ATLAS detector using 2015-2016 LHC proton-proton collision data. JINST. (2019) 14:P03017. doi: 10.1088/1748-0221/14/03/P03017

26. ATLAS Collaboration. Electron reconstruction and identification in the ATLAS experiment using the 2015 and 2016 LHC proton-proton collision data at $\sqrt{s}=13 \mathrm{TeV}$. Eur Phys J C. (2019) 79:639. doi: 10.1140/epjc/s10052-019-7140-6

27. ATLAS Collaboration. Muon reconstruction performance of the ATLAS detector in proton-proton collision data at $\sqrt{s}=13 \mathrm{TeV}$. Eur Phys J C. (2016) 76:292. doi: 10.1140/epjc/s10052-016-4120-y

28. Cacciari M, Salam GP, Soyez G. The anti- $k_{t}$ jet clustering algorithm. JHEP. (2008) 4:063. doi: 10.1088/1126-6708/2008/04/063

29. ATLAS Collaboration. Topological cell clustering in the ATLAS calorimeters and its performance in LHC Run 1. Eur Phys J C. (2017) 77:490. doi: 10.1140/epjc/s10052-017-5004-5

30. ATLAS Collaboration. Tagging and Suppression of Pileup Jets With the ATLAS Detector. ATLAS-CONF-2014-018 (2014). Available online at: https://cds. cern.ch/record/1700870

31. ATLAS Collaboration. Identification and rejection of pile-up jets at high pseudorapidity with the ATLAS detector. Eur Phys J C. (2017) 77:580. doi: 10.1140/epjc/s10052-017-5081-5

32. ATLAS Collaboration. Performance of $b$-jet identification in the ATLAS experiment. JINST. (2016) 11:P04008. doi: 10.1088/1748-0221/11/04/ P04008

33. ATLAS Collaboration. Optimisation of the ATLAS b-Tagging Performance for the 2016 LHC. ATL-PHYS-PUB-2016-012 (2016). Available online at: https:// cds.cern.ch/record/2160731

34. ATLAS Collaboration. Reconstruction, Energy Calibration, and Identification of Hadronically Decaying Tau Leptons in the ATLAS Experiment for Run-2 of the LHC. ATL-PHYS-PUB-2015-045 (2015). Available online at: https://cds. cern.ch/record/2064383

35. ATLAS Collaboration. Measurement of the Tau Lepton Reconstruction and Identification Performance in the ATLAS Experiment Using pp Collisions at $\sqrt{s}=13 \mathrm{TeV}$. ATLAS-CONF-2017-029 (2017). Available online at: https:// cds.cern.ch/record/2261772

36. ATLAS Collaboration. Performance of missing transverse momentum reconstruction with the ATLAS detector using proton-proton collisions at $\sqrt{s}=13 \mathrm{TeV}$. Eur Phys J C. (2018) 78:903. doi: 10.1140/epjc/s10052-018-6288-9

37. Elagin A, Murat P, Pranko A, Safonov A. A new mass reconstruction technique for resonances decaying to $\tau \tau$. Nucl Instrum Meth A. (2011) 654:481-9. doi: 10.1016/j.nima.2011.07.009

38. ATLAS Collaboration. Search for lepton-flavor-violating decays of the $Z$ boson into a $\tau$ lepton and a light lepton with the ATLAS detector. Phys Rev D. (2018) 98:092010. doi: 10.1103/PhysRevD.98. 092010

39. ATLAS Collaboration. Search for lepton-flavour-violating decays of the Higgs and $Z$ bosons with the ATLAS detector. Eur Phys J C. (2017) 77:70. doi: 10.1140/epjc/s10052-017-4624-0

40. ATLAS Collaboration. Search for the lepton flavor violating decay $Z \rightarrow e \mu$ in $p p$ collisions at $\sqrt{s}=8 \mathrm{TeV}$ with the ATLAS detector. Phys Rev D. (2014) 90:072010. doi: 10.1103/PhysRevD.90.072010

41. Collaboration TA. Observation of a new particle in the search for the Standard Model Higgs boson with the ATLAS detector at the LHC. Phys Lett B. (2012) 716:1-29. doi: 10.1016/j.physletb.2012.08.020

42. Collatoration TC. Observation of a new boson at a mass of $125 \mathrm{GeV}$ with the CMS experiment at the LHC. Phys Lett B. (2012) 716:30-61. doi: 10.1016/j.physletb.2012.08.021

43. Englert F, Brout R. Broken symmetry and the mass of gauge vector mesons. Phys Rev Lett. (1964) 13:321-3. doi: 10.1103/PhysRevLett.13.321

44. Higgs PW. Broken symmetries, massless particles and gauge fields. Phys Lett. (1964) 12:132-3. doi: 10.1016/0031-9163(64)91136-9

45. Higgs PW. Broken symmetries and the masses of gauge bosons. Phys Rev Lett. (1964) 13:508-9. doi: 10.1103/PhysRevLett.13.508

46. Higgs PW. Spontaneous symmetry breakdown without massless bosons. Phys Rev. (1966) 145:1156-63. doi: 10.1103/PhysRev.145.1156

47. Guralnik GS, Hagen CR, Kibble TWB. Global conservation laws and massless particles. Phys Rev Lett. (1964) 13:585-7. doi: 10.1103/PhysRevLett.13.585

48. Kibble TWB. Symmetry breaking in non-Abelian gauge theories. Phys Rev. (1967) 155:1554-61. doi: 10.1103/PhysRev.155.1554

49. ATLAS Collaboration. Search for lepton-flavour-violating decays of the Higgs boson in $\sqrt{s}=13 \mathrm{TeV} p p$ collisions with the ATLAS detector. Phys Lett B. (2019) 800:135069. doi: 10.1016/j.physletb.2019.135069 
50. ATLAS Collaboration. Search for the decays of the higgs boson $H \rightarrow e e$ and $H \rightarrow e \mu$ in $p p$ collisions at $\sqrt{s}=13 \mathrm{TeV}$ with the ATLAS detector. Phys. Lett. B. (2020) 135148. doi: 10.1016/physletb.2019.135148

51. Breiman L, Friedman J, Olshen R, Stone C. Classification and Regression Trees. New York, NY: Chapman \& Hall (1984).

52. Freund Y, Schapire RE. A decision-theoretic generalization of on-line learning and an application to boosting. J Comput Syst Sci. (1997) 55:119. doi: $10.1006 /$ jcss. 1997.1504

53. Friedman J. Stochastic gradient boosting. Comput Stat Data Anal. (2002) 38:367. doi: 10.1016/S0167-9473(01)00065-2

54. Chen T, Guestrin C. XGBoost: a scalable tree boosting system. In: Proceedings of the 22nd ACM SIGKDD International Conference on Knowledge Discovery and Data Mining. (2016). Available online at: https://dl.acm.org/citation.cfm? doid $=2939672.2939785$

55. Harnik R, Kopp J, Zupan J. Flavor violating Higgs decays. JHEP. (2013) 3:026. doi: 10.1007/JHEP03(2013)026

56. Tabanashi M. Review of Particle Physics. Phys Rev D. (2018) 98:030001. doi: 10.1103/PhysRevD.98.030001

57. ATLAS Collaboration. Search for lepton-flavour-violating $H \rightarrow \mu \tau$ decays of the Higgs boson with the ATLAS detector. JHEP. (2015) 11:211. doi: 10.1007/JHEP11(2015)211

58. Aubert B, BaBar Collaboration. Searches for Lepton Flavor Violation in the Decays $\tau^{ \pm} \rightarrow e^{ \pm} \gamma$ and $\tau^{ \pm} \rightarrow \mu^{ \pm} \gamma$. Phys Rev Lett. (2010) 104:021802. doi: 10.1103/PhysRevLett.96.041801

59. ATLAS Collaboration. A Search for the Dimuon Decay of the Standard Model Higgs Boson in pp Collisions at $\sqrt{s}=13 \mathrm{TeV}$ With the ATLAS Detector. ATLAS-CONF-2019-028 (2019). Available online at: http://cds.cern. $\mathrm{ch} /$ record/2682155

60. MEG Collaboration. Search for the lepton flavour violating decay $\mu^{+} \rightarrow \mathrm{e}^{+} \gamma$ with the full dataset of the MEG experiment. Eur Phys J C. (2016) 76:434. doi: 10.1140/epjc/s10052-016-4271-x

61. Bellgardt U, SINDRUM Collaboration. Search for the decay $\mu^{+} \rightarrow e^{+} e^{+} e^{-}$. Nucl Phys B. (1988) 299:1-6.

62. Bertl W, SINDRUM II Collaboration. A search for $\mu$-e conversion in muonic gold. Eur Phys J C. (2006) 47:337-46. doi: 10.1140/epjc/s2006-02582-x

63. ATLAS Collaboration. Search for lepton-flavor violation in different-flavor, high-mass final states in $p p$ collisions at $\sqrt{s}=13 \mathrm{TeV}$ with the ATLAS detector. Phys Rev D. (2018) 98:092008. doi: 10.1103/PhysRevD.98.092008

64. ATLAS Collaboration. Search for high-mass dilepton resonances in $p p$ collisions at $\sqrt{s}=8 \mathrm{TeV}$ with the ATLAS detector. Phys Rev D. (2014) 90:052005. doi: 10.1103/PhysRevD.90.052005

65. Choudalakis G. On hypothesis testing, trials factor, hypertests and the BumpHunter. arXiv.org [Pre-print]. arXiv:1101.0390 (2011).

66. Barbier R, Berat C, Besancon M, Chemtob M, Deandrea A, Dudas E, et al. R-parity violating supersymmetry. Phys Rept. (2005) 420:1-202. doi: 10.1016/j.physrep.2005.08.006

67. Arkani-Hamed N, Dimopoulos S, Dvali GR. The hierarchy problem and new dimensions at a millimeter. Phys Rev B. (1998) 429:263-72. doi: 10.1016/S0370-2693(98)00466-3

68. Randall L, Sundrum R. A large mass hierarchy from a small extra dimension. Phys Rev Lett. (1999) 83:3370-73. doi: 10.1103/PhysRevLett.83.3370

69. Gunion JF, Vega R, Wudka J. Higgs triplets in the standard model. Phys Rev D. (1990) 42:1673-91. doi: 10.1103/PhysRevD.42.1673
70. Senjanovic G, Mohapatra RN. Exact left-right symmetry and spontaneous violation of parity. Phys Rev D. (1975) 12:1502-5. doi: 10.1103/PhysRevD.12.1502

71. Bhupal Dev PS, Mohapatra RN, Zhang Y. Displaced photon signal from a possible light scalar in minimal left-right seesaw model. Phys Rev D. (2017) 95:115001. doi: 10.1103/PhysRevD.95.115001

72. Akeroyd AG, Aoki M. Single and pair production of doubly charged Higgs bosons at hadron colliders. Phys Rev D. (2005) 72:035011. doi: 10.1103/PhysRevD.72.035011

73. Lazarides G, Shafi Q, Wetterich C. Proton lifetime and fermion masses in an SO(10) model. Nucl Phys B. (1981) 181:287-300. doi: 10.1016/0550-3213(81)90354-0

74. Huitu K, Maalampi J, Pietil A, Raidal M. Doubly charged Higgs at LHC. Nucl Phys B. (1997) 487:27-42. doi: 10.1016/S0550-3213(97)87466-4

75. ATLAS Collaboration. Search for doubly charged Higgs boson production in multi-lepton final states with the ATLAS detector using protonproton collisions at $\sqrt{s}=13 \mathrm{TeV}$. Eur Phys J C. (2018) 78:199. doi: 10.1140/EPJC/S10052-018-5661-Z

76. ATLAS Collaboration. Search for anomalous production of prompt samesign lepton pairs and pair-produced doubly charged Higgs bosons with $\sqrt{s}=8 \mathrm{TeV} p p$ collisions using the ATLAS detector. JHEP. (2015) 3:041. doi: 10.1007/JHEP03(2015)041

77. Cerri A, Gligorov VV, Malvezzi S, Martin Camalich J, Zupan J. Report from Working Group 4: opportunities in flavour physics at the HLLHC and HE-LHC. CERN Yellow Rep. Monogr. (2019) 7:867-1158. doi: 10.23731/CYRM-2019-007.867

78. Cepeda M, Gori S, Ilten P, Kado M, Riva F. Report from Working Group 2: Higgs physics at the HL-LHC and HE-LHC. CERN Yellow Rep. Monogr. (2019) 7:221-584. doi: 10.23731/CYRM-2019-007.221

79. Decamp D. Searches for new particles in $Z$ decays using the ALEPH detector. Phys Rept. (1992) 216:253-340. doi: 10.1016/0370-1573(92) 90177-2

80. Abreu P. Search for lepton flavor number violating Z0 decays. Z Phys C. (1997) 73:243-51. doi: 10.1007/s002880050313

81. Adriani O. Search for lepton flavor violation in $\mathrm{Z}$ decays. Phys Lett B. (1993) 316:427-34.

82. Akers R. A Search for lepton flavor violating Z0 decays. Z Phys C. (1995) 67:555-64.

83. Cid Vidal X, D’Onofrio M, Fox PJ, Torre R, Ulmer KA. Report from Working Group 3: Beyond the Standard Model physics at the HL-LHC and HE-LHC. CERN Yellow Rep. Monogr. (2019) 7:585-865. doi: 10.23731/CYRM-2019-007.585

Conflict of Interest: The authors declare that the research was conducted in the absence of any commercial or financial relationships that could be construed as a potential conflict of interest.

Copyright (๑) 2020 Davidek and Fiorini. This is an open-access article distributed under the terms of the Creative Commons Attribution License (CC BY). The use, distribution or reproduction in other forums is permitted, provided the original author(s) and the copyright owner(s) are credited and that the original publication in this journal is cited, in accordance with accepted academic practice. No use, distribution or reproduction is permitted which does not comply with these terms. 